\title{
On the Fragmentation of Explosively-driven Plastic/steel Layered Cylinders
}

\author{
EUGEN TRANA, FLORINA BUCUR, ADRIAN N. ROTARIU* \\ Military Technical Academy Ferdinand I, 39-49 George Cosbuc Av., 040531, Bucharest, Romania
}

\begin{abstract}
While over the past century fairly extensive work has been carried outon explosively-driven metallic envelope fragmentation, as the development of explosive materials and warhead configurations continued, the issue under consideration is still topical. The current paper aims to investigate the fragmentation characteristics of explosively-driven steel cylinders when double layer envelopes are used. Mott's approach was modified in order to account for such particular configuration (layered envelope). Several tests in a simplified type procedure were performed aiming to confirm the theoretical approach.
\end{abstract}

Keywords: layered envelope, detonation, fragmentation

Whether considering pressurize gas recipients critical malfunctions, combustion chambers malfunctions, security glass shatter, car crash events, high velocity impacts events or artillery shells detonation, a special interest must be paid to the likely possible or intended fragmentation feature. In fact, the same interest can be extended if required even to astronomical events as meteor or comet collapses shown by Grady [1].

Actually, based on a simple literature survey one can say that the interest in solid bodies' natural fragmentation process, over the last century, proved to be a fairly debated topic with direct implication both in civil and military applications.

Although, several empirical models on military applications were available starting with 1920's (J ustrow or Yulovsky, for example [2]), the first serious reference on fragmentation as a scientific approach can be traced back to 1933 when puzzled by the observed particular characteristic of coal fragmentation process, Rosin and Rammler proposed a mathematical approach with regard to the number of fragments and the distribution of fragment sizes related with the natural fragmentation [3].

After this firstserious contribution, in the following years several other scientists [4-10] contributed also in revealing both the physical and statistical concepts that lie behind the fragmentation phenomenon.

Basically, as a common feature, all fragmentation models estimate the fragment characteristic length or/and the total number of fragments or, in the case of some empiric models, the number of fragments with mass exceeding a certain value.

It is worth noticing that the majority of previouslymentioned models and methodologies were stated considering the case of single layer envelope.
However, due to the inherent evolution of warheads structures, double layer configurations became quite customary. Since usually the interior envelope has either a pre-fragmented or an inert nature became of special interest the natural fragmentation of the exterior envelope.

For the present study an investigation on the fragmentation of explosively-driven double layer cylinders is carried out. In order to facilitate the identification of exterior envelope fragments a testing configuration Plastic foil/Steel for the double layer cylinder will be adopted.

\section{Experimental part}

Materials and test configuration

For the experimental approach a simplified procedure built on two test configurations like the ones depicted in figure 1 were set-up.

The tests were run on High Explosive (HE)/Plastic foil/ Steel envelope specimens (fig. 2). For the HE a $37^{-} \mathrm{mm}$ diameter and $65 \mathrm{~mm}$ height cylindrical HITEX (plastic explosive with 91\% RDX and 9\% binder) charge was used.

Several $25 \mu \mathrm{m}$ PVC foil layers with a total mass of 0.009 $\mathrm{kg}$ and $3 \mathrm{~mm}$ thickness were wrap-up around the explosive. As metallic envelope a $0.275 \mathrm{~kg} \mathrm{S355)} 2 \mathrm{H}$ (OL 52) steel grade cylinder $(\Phi=43 \mathrm{~mm}, \Phi=51 \mathrm{~mm}, \mathrm{~h}=65 \mathrm{~mm})$ was used for the tested items. According to supplier sheet, S355) $2 \mathrm{H}$ presented a $396 \mathrm{~N} / \mathrm{mm}^{2}$ yield stress point, a 537 $\mathrm{N} / \mathrm{mm}^{2}$ ultimate stress value along with a $31 \%$ elongation limit.

To initiate HITEX detonations, classic electrical blast caps were used. The test program included three trials for each experimental set-up.

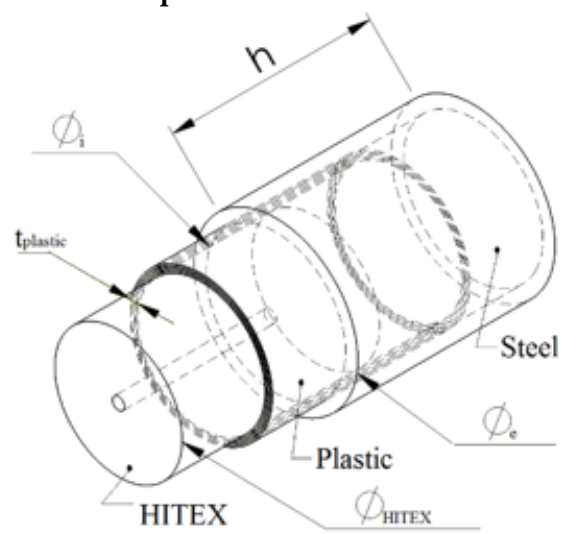

Fig. 2. Exploded view test specimens geometry

Fig. 1. Experimental set-up

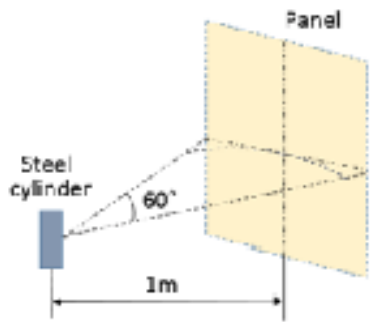

b

*email:rotariu@mta.ro 
One set of tests involved the use of water recipients at $0.6 \mathrm{~m}$ from the detonation site to decelerate and retain the generated steel fragments with a special emphasis on effective fragments (fragments with mass above 1gram) (fig. 1a). By this manner the time consuming standard test involving huge quantities of sand was replaced. The other set of tests was design to investigate the total number of fragments formed on cylinder detonation by recording for a $60^{\circ}$ arch the fragment impact prints on a $1.16 \times 2 \mathrm{~m}$ wood panel deployed at exactly $1 \mathrm{~m}$ (fig. 1b).

\section{Results and discussions}

As mentioned previously the proposed testmethodology was design to study the double layer cylinders fragmentation by investigating the total number of fragments and the number of effective fragments.

Typical example of recovered effective fragments and relevant experimental data are synthetized in figure 3 and table 1.

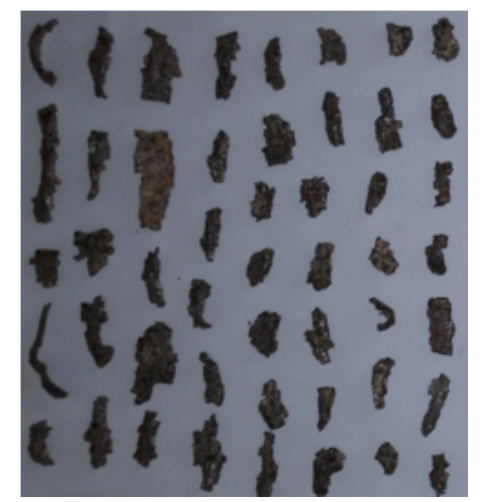

Fig. 3. Typical examples of recovered effective fragments

Table 1

TEST RESULTS

\begin{tabular}{|c|c|c|}
\hline Test no. & $\begin{array}{c}\text { Recovered average } \\
\text { effective fragments } \\
\text { number }\end{array}$ & $\begin{array}{c}\text { Counted average number } \\
\text { of impacts for } 60^{\circ} \text { arch }\end{array}$ \\
\hline 1 & 63 & 181 \\
\hline 2 & 60 & 182 \\
\hline 3 & 63 & 180 \\
\hline Average & 62 & 181 \\
\hline
\end{tabular}

In order to correlate and extend the experimental findings for the double layer cylinder fragmentation a theoretical approach was considered. As starting point Mott's model was adopted having in mind that this is the most famous theoretical based model used in shell fragmentation analysis. The mathematical relations used by Mott points to the following equations:

$$
\begin{aligned}
& M_{A}=B t^{\frac{5}{6}} d^{\frac{1}{3}}\left(1+\frac{t}{d}\right) \\
& N_{0}=\frac{M}{\bar{m}} \\
& \bar{m}=2 \mu \\
& \mu=M_{A}^{2}(1) \\
& N_{x}=N_{0} e^{-\left(\frac{x}{M_{A}^{2}}\right)^{1 / 2}}
\end{aligned}
$$

where: $M$ stands for a mass proportionality factor, $B$ is a constant of the explosive, $t$ indicates the cylinder thickness, $d$ is the cylinder interior diameter, $M$ denotes cylinder metal mass, $N_{0}$ is the total number of fragments, $\bar{m}$ is distribution mean (fragment average mass), $\mu$ stands for a distribution factor, $N_{x}$ indicates the number of fragments with a mass above $x$.

The equations refers to the classic single layer cylinder as depicted in figure 4.

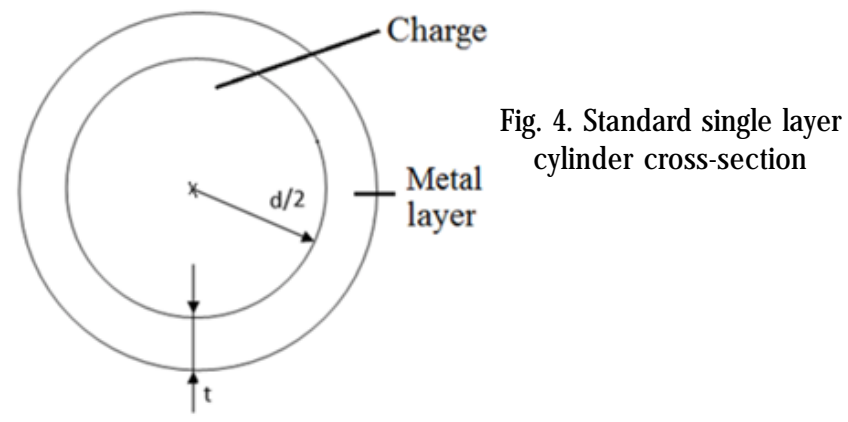

Considering Mott's equations, one can conclude that it is entirely based on $M_{A}$ expression. As pointed out in [11] the mass proportionality factor $\left(M_{A}\right)$ was stated for a single layer envelope that assumes a direct contact with the charge. Thereby, any attempt to use equations (1) to (5) for a different type of configuration (double layer envelope - fig. 5) should first deal with the correct amendment of equation (1). Due to the particular studied geometry the effort will be focused on deriving a new expression for the mass proportionality factor that will include the existence of an intermediary layer.

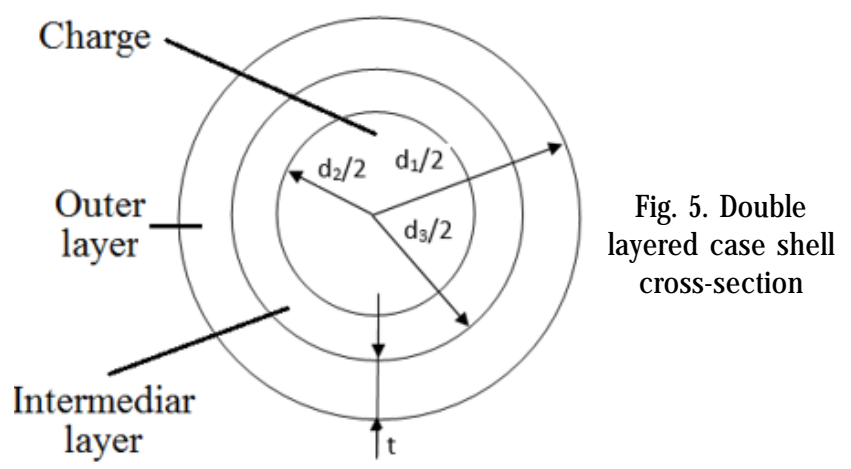

As indicated by Mott [11], the basic $M_{A}$ form (equation 6 ) is related to the cylinder cross-section dimension.

$$
M_{A}=K r^{\frac{2}{3}} t^{\frac{1}{2}} v^{-\frac{2}{3}}
$$

where: $r$ and $t$ stand for the casing radius and thickness at the moment of break-up, $v$ is the radial velocity of the envelope ring at the same moment and $K$ indicates a constant that includes parameters like density and fracture specific energy.

Basically, the same equation (6) can be applied to the particular case of an extra inert layer placed between charge and case changing accordingly the radial velocity.

Additionally to equation (6), the energy conservation law (equation (7)) will also be considered as indicated by Grady [10]:

$$
\frac{1}{2} M_{0} v^{2}(r)+\frac{1}{2} M_{1} v^{2}(r)+\omega U(r)=\omega E
$$

where: $M_{0}$ is the outer layer mass (steel mass), $M_{1}$ stands for the intermediar layer (plastic or aluminium, for example), $\varepsilon$ is the charge mass, $U(r)$ indicates the specific compressional energy of the expanding explosive products and $E$ is a material constant that expresses the compressional energy at the initial cylinder radius. 
Equation (7) as highlighted by Grady accounts for envelope speed-up but fails to consider the kinetic energy of the expanding explosive products and solid envelope deformation energy requirement. Nevertheless, since equation (7) was the original Mott's approach one will continue with that particular equation.

Considering the extra layer mass as a percentage of the outer layer $\left(\grave{\mathrm{e}}=\mathrm{M}_{1} / \mathrm{M}_{0}\right)$ one can write:

$$
v^{2}(r) \frac{M_{0}(1+\theta)}{\omega}=2 E\left(1-\frac{U(r)}{E}\right)
$$

or

$$
v^{2}(r) \frac{d_{1}^{2}-d_{3}^{2}}{d_{2}^{2}}(1+\theta)=2 E\left(1-\frac{U(r)}{B}\right) \frac{\rho_{C}}{\rho_{m}}
$$

with: $\rho_{c}$ and $\rho_{m}$ charge and outer layer material density.

Using the same assumption as Mott regarding $u_{0}$ concept, $u_{0}=\sqrt{2 E}$ ( $u_{0}$ is considered to be a property only of the explosive-metal system) equation (9) became:

$$
\frac{1}{v^{2}}=\frac{8}{u_{0}^{2}} \frac{r t}{d_{2}^{2}}(1+\theta)
$$

where: $r=\frac{d_{1}+d_{3}}{4}$ and $t=\frac{d_{1}-d_{3}}{2}$.

Including r expression and equation (10), equation (6) can be rewritten as follows:

$$
M_{A}=K\left(\frac{1}{u_{0}^{2}}\right)^{\frac{1}{3}}(1+\theta)^{\frac{1}{3}} \frac{2 r t^{\frac{5}{6}}}{d_{2}^{\frac{2}{3}}}
$$

or considering Mott's constant $C\left(0.0531 \mathrm{lb}^{1 / 2} \mathrm{in}-7 / 6\right.$ the constant of RDX as indicated in [12] or $2.597 \mathrm{~kg}^{1 / 2} \mathrm{~m}^{-7 / 6}$ in international units)

$$
M_{A}=C(1+\theta)^{\frac{1}{3}} \frac{2 r t^{\frac{5}{6}}}{d_{2}^{\frac{2}{3}}}
$$

Since $r t=c s t$ as Mott proposed, in equation (12) the initial values for $r$ and $t$ will be used.

Considering equation (12) for Mott's model, a total number of 1110 fragments with 64 effective fragments is calculated.

Compared against each other, the theoretical and experimental results on total number of fragments and effective fragment number (fig. 6) display a good correlation (1110 vs 1086 respectively 64 vs 62).

The differences observed in figure 6 could be assigned to both a natural data dispersion and to a less perfect effective fragment collecting procedure. In fact, based on the results, the proposed fragment collecting procedure even expedite clearly misses the capability to offer a complete image on fragments mass distribution.

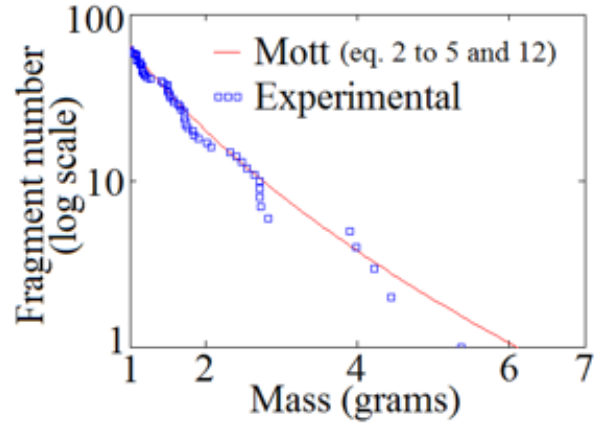

Fig. 6. Experimental vs theoretical effective fragment findings

\section{Conclusions}

The paper aims to investigate the fragmentation pattern of the exterior metal envelope in a particular double layer configuration (plastic/metal). As underlined in the paper, the fragmentation phenomenon of the particular studied configuration can be investigated both theoretically and experimentally. The theoretical approach can be pursued using Mott's algorithm.

The decision to use PVC foils was based on the necessity to have an inhert intermediary layer with a higher plasticity than the S355) 2H layer.

Although raw, the proposed testing methodology proved itself to be promising. The theoretical and experimental findings are correlated.

Acknowledgement: The current work was supported by a grant of the Romanian National Authority for Scientific Research and Innovation, CNCS/CCCDI - UEFISCDI, project number PN-III-P2-2.1PED-2016-0057, within PNCDI III.

\section{References}

1. GRADY, D.E., Int.J.Fract. 163, 2010, pp. 85

2. CRANZ, C., Zweiter band - Innere ballistic, Springer-Verlag Berlin Heidelberg $\mathrm{GmbH}, 1926$

3. ROSIN, P., RAMMLER, E., Journal of the Institute of Fuel, 7, 1933, pp. 29

4. LIENAU, C.C., J ournal of the Franklin Institute, 221, 1936, pp. 485 5. WEIBULL, W. A., Proceedings of the Ingeniors Vetenskapsakad. Handlingar, 1939, pp. 151

6. SCHUHMANN E.V., AIME Technical Publication, 1189, 1941, pp. 1

7. MOTT, N.F., Proceedings of the Royal Society of London Series AMathematical and Physical Sciences, 189 (1018), 1947, pp. 300

8. GILVARRY, J.J., J ournal of Applied Physics, 32(3), 1961, pp. 391

9. SHOCKEY, D.A., CURRAN, D.R., SEAMAN, L., PETERSEN, C.F., International J ournal of Rock Mechanics and Geomechanics Abstracts, 11, 1974, pp. 303

10. GRADY, D.E., Fragmentation of rings and shells. The legacy of N.F. Mott, Springer-Verlag Berlin Heidelberg, 2006

11. MOTT, N.F., A.O.R.G. Memorandum no. 24, 1943

12. COOPER, P.W., Explosives Engineering, Wiley-VCH Inc. New-York, 1996

Manuscript received: 13.09 .2018 